\title{
Prospective data registration and clinical trials for particle therapy in Europe
}

Langendijk $J A^{1}$, Orecchia $R^{2}$, Haustermans $K^{3}$, Zips D4, Balosso $~^{5}$, Lacombe $D^{6}$, Lievens $Y^{7}$, Weber $D C^{8}$,
Grau $C^{9}$, Troost $E G C^{10}$.

1) Department of Radiation Oncology, University of Groningen, University Medical Center Groningen, The Netherlands. Electronic address: j.a.langendijk@umcg.nl.

2) European Institute of Oncology, Milan, Italy.

3) Department of Oncology, Laboratory of Experimental Radiotherapy, KU Leuven - University of Leuven \& Department of Radiation Oncology, University Hospitals Leuven, Belgium.

4) Department of Radiation Oncology, University of Tübingen, Germany.

5) Department of Radiation Oncology and Medical Physics, University Hospital of Grenoble Alpes (CHU-GA), France; France HADRON National Research Infrastructure, IPNL Lyon, France; University Grenoble Alpes, France.

6) European Organisation for Research and Treatment of Cancer (EORTC), Brussels, Belgium.

7) Radiation Oncology Department, Ghent University Hospital and Ghent University, Belgium. 8) Center for Proton Therapy, Paul Scherrer Institute, ETH Domain, $\mathrm{CH}-5232$ Villigen, Switzerland; Department of
Radiation Oncology, University Hospital of Bern, Switzerland.

9) Department of Oncology, Aarhus University Hospital, Denmark.

10) OncoRay - National Center for Radiation Research in Oncology, Faculty of Medicine and University Hospital Carl Gustav Carus, Technische Universität Dresden, Helmholtz-Zentrum Dresden Rossendorf, Germany; Department of Radiotherapy and Radiation Oncology, Faculty of Medicine and University Hospital Carl Gustav Carus, Technische Dresden, Germany.

\section{Abstract}

The overarching aim of work package 1 of the European Proton Therapy Network (EPTN) is to create a firm basis for evidence-based particle therapy at the European level. To achieve this, this work package will set up a worldwide unique prospective data registration programme for nine different tumour sites. Such programme will provide more insights into the current practice across all European particle therapy centres and into the results of particle therapy with regard to radiation-induced toxicity and efficacy in terms of local control and survival.

More importantly, prospective data registration provides major opportunities to continuously improve the quality of particle therapy, by defining bench marks, to identify best practices that may learn others to improve quality of particle therapy, to synchronize selection criteria and to create more homogeneous patient cohorts to evaluate results, which is particularly important in rare tumours.

This will be supported by EORTC through existing and new IT-infrastructure for data collection in different formats next to QA-platforms.

In addition, work package 1 will define the requirements for high quality clinical trials in order to enhance high quality clinical trial proposals and determine alternative methods for RCT, such as the model-based approach. 
Particle therapy offers great opportunities to further broaden the therapeutic ratio of radiotherapy by either decreasing the dose to normal tissues while the target dose remains equivalent or by target dose escalation without further excess dose to the normal tissues. However, there is widespread discussion regarding lack of evidence for proton treatment for a wide range of indications. Even for the most widely accepted conditions, paediatric tumours, issues remain as to whether superiority of protons over photons has sufficiently been shown [1]. Reducing dose to normal tissues and organs outside of target areas evidently is the key feature of protons versus photons, but translation of these reductions into clinically relevant benefits has still not been demonstrated consistently, including biological issues such as variable relative biological effectiveness [2].

Therefore, to enhance evidence-based particle therapy, EPTN decided to establish a dedicated work package (work package 1 ) to create a firm basis for evidence-based particle therapy at a European level. To this purpose, the following objectives were defined:

1. to determine the content of uniform prospective data registration programmes at a European level for the most common tumour types treated with particle therapy;

2. to setup an IT infrastructure that can support the model-based approach at a European level by harmonizing data acquisition, making data Findable, Accessible, Interoperable and Reusable (FAIR) and linking data from different sources and centres [9].

3. to identify the methodological issues related to phase I and II studies as well as to RCTs comparing photons with particles and to define general guidelines for the design of clinical trials to overcome these issues; to establish an Expert Committee to advice and support researchers in Europe in the design of clinical trials in particle therapy.

The aim of this paper is to further explore the background of these objectives and to briefly discuss the strategy to further enhance evidence-based introduction of particle therapy on a European level.

\section{Prospective data registration}

The general idea is to create a firm prospective data registration programme for all patients treated in European particle therapy centres which is considered important for a number of reasons:

First, major differences exist between European proton therapy centres on criteria for patient selection, resulting in major heterogeneity of patient populations and eventual outcome. Prospective data collection of all patients treated with proton therapy in Europe will provide essential information on patient mix and outcome and may serve as a basis to discuss and harmonize selection criteria for
particle therapy in order to create more homogeneous patient particle therapy in order to create more homogeneous patient populations. In this way, evaluation of the efficacy of particle therapy in well-defined patient populations can be accelerated, which is particularly useful for relatively rare tumours, such as base of skull tumours and paediatric malignancies. Such data may also be used as to generate hypotheses for and to design future clinical
trials.

Second, particle therapy is a relatively new radiation modality and needs to undergo some form of quality assurance. There is major variability in the performance between centres due to differences in delivery and treatment planning equipment, differences in standard operation procedures (e.g. the use of image-guidance and plan adaptation) and differences in expertise, experience, composition and treatment philosophies of the health care teams involved. Multicentre uniform prospective data collection provides unique opportunities to define benchmarks and to identify best practices. This information can be used to continuously improve the quality of particle therapy on a European level. 
Third, prospective data collection is the hall mark of the model-based approach, an evidence-based methodology introduced in the Netherlands for both patient selection and clinical validation of proton therapy, which could serve as an alternative for randomized controlled trials (RCTs), which are still considered the gold standard of evidence-based medicine.

In this regard, it should be emphasized that there is no doubt that an RCT is the most appropriate study design when the main goal is to increase treatment efficacy in terms of local control or survival by target dose escalation beyond the dose considered current standard. For such an application of particles, not only the effect of dose escalation on tumour control must be explored, but also the risks of consequent dose escalation to the normal tissues nearby the target beyond levels that are normally administered. In addition, when the biological effect of particles is possibly different from currently used photons, e.g. higher RBE when using carbon ions, RCTs are required not only when the primary objective is to improve local control, but also when particles are applied to reduce radiation-induced side effects.

It is expected that in most cases, protons will be applied to prevent radiation-induced side effects and/or induction of secondary tumours. In 2009, the Dutch Health Council produced an extensive report on the expected indications for proton therapy and concluded that around 5-10\% of all patients currently treated with radiotherapy would benefit from protons, and that most (85\%) of them will be treated with particles to prevent radiation-induced side effects and/or secondary tumour induction [3]. For the validation of radiation technologies primarily aiming at reduction of side effects, there is a growing awareness that equating evidence-based medicine with RCTs is an undue simplification and that other methodologies, such as the model-based approach, are available and need further exploitation $[3,4]$.

Irrespective of the research question, the design of RCTs when comparing two different radiation technologies may be subject to methodological difficulties and pitfalls as well. Therefore, it is important not only to identify and address these difficulties but also to define how they can be prevented. In addition, the definition of minimal requirements for the design of RCTs comparing photons with particles is desperately needed to guarantee generalizability of results and eventual proper translation into routine clinical practice.

In the Netherlands, an alternative methodology has been developed to select patients for proton therapy and to validate the benefit of protons over photons: the so-called model-based approach (MBA). The MBA is an evidence-based methodology designed to yield evidence for a more rational selection of patients who would most likely derive clinically relevant benefits from particle therapy in terms of prevention of radiation-induced side effects $[5,6]$. The rationale behind model-based selection is that particle therapy will only lead to broaden the therapeutic window by decreasing toxicity, when three essential requirements are met: (1) the dose to the target is equivalent to photons and considered current standard; (2) normal tissue sparing can be obtained with particles compared to photons ( $\Delta$ Dose), and (3) $\Delta$ Dose results in a clinically significant lower complication risk (or else lower normal tissue complication probability $(\triangle N T C P)$ ). It should be stressed that transforming dose into complication risk requires multivariable NTCP-models including non-dosimetric features (e.g., patients' age, concomitant chemotherapy) and that therefore a decrease of dose will not always translate into a relevant decrease of complication risks.

The key research agenda for the near future should therefore be to validate this thesis by attempting to falsify the hypothesis that NTCP reduction leads to less toxicity, which is the main principle of modelbased validation. In addition, it is very likely that NTCP-models need continuous updating and adjustments due to differences in patient mix and technological evolvements $[7,8]$. For this purpose, 

uniform prospective data registration at a European level of all patients treated with proton therapy
is essential.

Our main priority is therefore to establish uniform prospective data registration programmes on a European level for the most common tumour types treated with particle therapy. Therefore, nine tumour-specific sub-tasks were established for patient groups that are frequently treated with particle therapy, including tumours of the central nervous system (CNS), head and neck, breast, lung, oesophagus, lymphoma, sarcoma, prostate, and paediatric cancer. Next to a generic assessment that applies for all patients irrespective of tumour site, these sub-tasks are defining the data sets for each
tumour site.

As mentioned earlier, the main objective of the prospective data registry is to get more information on the characteristics of patients treated with particles and to get more insight into the most relevant outcome measures. There is consensus that such registry can only be informative and successful when in principle all European particle centres will be able to participate and when patient accrual and compliance to the programme is high. However, reality is that resources for data registries are generally limited. Therefore, EPTN decided to define different levels of data registries to on the one hand ensure participation of all centres and, at the other hand, offer opportunities to collect more comprehensive or detailed data by a limited number of centres (Table 1).

The level I dataset is the minimal dataset that is mandatory for all EPTN centres. The first version of the generic level I dataset that applies to all patients irrespective of tumour site is outlined in Table 2 next to an example of a tumour-specific level I dataset. Typical examples of level I items are baseline characteristics like gender, age, primary tumour site, stage, and other treatment modalities used. Level I also contains PROMs such as the EORTC QLQ-C30 to assess quality of life at baseline and at predefined time points during follow up. Finally, items related to tumour control and survival are considered level I as well. For breast, prostate and lung cancer, the standard sets as defined by the International Consortium for Health Outcomes Measurement (ICHOM) is a good starting point (http://www.ichom.org/medical-conditions).

As particle therapy is more expensive than photon therapy, which is an important issue in many discussions on particle therapy, varying from individual patient level to discussions at the national level, there was consensus that data supporting the economic discussion, such as utility values measured through EQ-5D, should also be included [10].

Level II datasets are optional and are organized into predefined packages around a certain theme. An example of a level II dataset could be a more comprehensive set of items related to radiation-induced cardiac toxicity after proton therapy for breast cancer, in which risk factors for major cardiac events at baseline and major cardiac events during follow up are added to the minimal level I dataset. The collection and registration of dose volume histogram (DVH) data and/or the creation of an EPTN radiation dose registry containing planning-CT with RT-DICOM-struct and RT-DICOM-dose data can also be part of the level II dataset. An important feature of level II as compared to level III items is that no additional diagnostic procedures are required apart from physician-rated toxicity assessment
and/or PROMs.

Level III dataset are also optional and include assessments, for which additional diagnostic procedures, beyond what is generally considered standard of care, are needed. These assessments are generally part of clinical observational or intervention studies, and require formal medical ethical approval. Level III datasets are always combined with the level I generic and level I tumour-specific dataset and can be combined with a level II dataset. In the example of cardiac toxicity in breast cancer patients, level III items may include echocardiography and cardiac MRI. 
The 3-level approach has several advantages. First, defining a limited dataset (level I) requiring limited resources provides the highest probability that all European particle centres can participate, which will provide unique and valuable information on patient mix and different kinds of outcome. Second, level II items can be easily added by a limited number of centres with a similar patient mix to get more insight in the effects of particle therapy in specific patient groups. Our experience is that patients are more easily willing to complete additional questionnaires when added to those that are considered standard. In addition, when PROM assessment logistics are already in place, it is less burdensome for centres to just add one or more questionnaires or toxicity assessments. Third, if level I either or not combined with level II datasets are established, it is relatively easy to integrate this in formal RCTs or the concept of cohort multiple RCTs [11]. Finally, as prospective data registration is the backbone of the model-based approach combining level I with level II datasets will also allow for model-based

\section{Vision and scope on clinical trials}

During the EPTN meeting in April 2016, the vision and scope for clinical trials in the EPTN were discussed and were formulated as follows:

- Emphasis should be on performing high quality clinical trials with properly selected patients and using relevant, validated clinical endpoints.

- A small number of pivotal RCTs are urgently needed. However, we need to develop, test and validate alternative evidence-based methodologies (e.g. "cohort multiple RCTs").

- Model-based selection (as predictive biomarker) is a useful concept for NTCP-based clinical trials, and this concept should later be extended to incorporate Tumor Control Probability
(TCP) as well.

- European centres must join forces to create such trials and evidence soon.

- Trials involving state-of-the-art photon RT are welcome, as particle therapy should be an integral component of radiation oncology.

- European trials should be open to accredited centres with expertise and relative high numbers who wish to collaborate.

- Prospective collection of high quality data for patients treated with proton therapy outside of clinical trials (using common ontology and data collection forms).

- Uniform guidelines for target and organ at risk delineation, as well as guidelines on dose constraints.

- There is a need to develop an IT-infrastructure and European QA platform, not only for particle therapy trials, but also for prospective data registries.

\section{Prospective data registries}

\section{IT-infrastructure/QA platform}

Particle therapy planning and delivery have changed substantially over the last three decades, starting with the introduction of passive scattered protons in the 1980 s, followed by pencil beam scanned (PBS) PT in the 1990s, to image-guided and adaptive PT currently, with a substantial complexity in the planning and delivery process. The evaluation of these potentially error-prone techniques in clinical trials with PT require careful and thorough quality assurance (QA) programmes, which the EORTC has pioneered since the 1990s. Recently, the EORTC published the results of a facility questionnaire 
assessment, representing level I of QA, for European particle centres [12]. QA in radiotherapy in general and particle treatment in particular is defined by all of those procedures that ensure consistency of the radiation prescription and the safe fulfilment of that prescription with regard to the dose to the cancer and organs at risk (OARs), minimization of exposure of personnel, and patient monitoring aimed at determining the results of treatment [13]. A QA programme is also aimed at defining the range of acceptable deviations, detecting potential causes for larger deviations, and developing mechanisms of action for correction and prevention of these deviations [14]. The goal is to reduce variability and uncertainties related to the different steps of treatment planning and actual patient irradiation, including but not limited to patient positioning, correct beam delivery output and precise dose delivery to the cancer. Specific considerations of QA for particles in a multicentre research setting include promotion of consistency between particle centres, ensuring sites meet minimum technical and personnel requirements for a given clinical trial, ensuring accuracy and integrity of data and importantly to assess inter-patient and interinstitutional variation to name a few concerns. The EORTC will bring substantial expertise in this field and offers a QA platform and IT-support tested in numerous prospective clinical trials assessing the efficacy of radiotherapy.

\section{Trial methodology and support}

Next to the setting up of a particle data registry and IT-infrastructure, it is of major importance to identify the methodological issues related to phase I, II and III studies and, in addition, to define guidelines for the design of clinical trials on particle therapy. Comparing different radiation technologies is challenging and numerous methodological issues related to clinical studies on particle therapy must be addressed. In respect to these challenges, the EORTC will provide support to the EPTN investigators by providing access to imaging or radiotherapy QA platforms as mentioned in the previous paragraph, statistical design expertise and databases that will be able to include information from multiple sources, including but not limited to diagnostic/molecular imaging, particle radiation therapy parameters, prospective QA assessments, and biological samples [15].

To advice and support clinical researchers in Europe, a platform of experts will be established. To this end, an invitational conference will be organized with experts in the field of particle therapy (radiation oncologists and medical physicists), methodology, epidemiology and statistics. Based on the outcome of this conference, a checklist with minimal requirements and quality points will be created to improve consistency between trials. This checklist can then be used to review future studies and trial protocols. In this regard, this embarks on the vision and scope as previously discussed within EPTN and to embark on the work already done by the Union of Light Ion Centres in Europe (ULICE) (http://ulice.web.cern.ch/ULICE/cms/index.php?file=results), in particular JRA 2.2 (review of existing protocol structure in large clinical research organization), JRA 2.3 (description of tasks with a proposal for potential structures for clinical research) and JRA 2.6 (implementation, testing and evaluation of the structure with typical "cases" for research and development).

Finally, an Expert Committee (EC) will be founded with experts in the field that can be consulted in the design of future clinical studies on particle therapy. The EC will also be responsible for setting up meetings in which future studies can be discussed. It should be noted that (1) ESTRO/EPTN is not going to conduct clinical trials but intends to use existing platforms and organizations, and (2) external funding is needed to run such trials. 


\section{Conclusion}

The main objective of EPTN WP1 is to establish a uniform prospective data registration programme for all patients treated with particle therapy in Europe. This will be supported by EORTC through existing and new additional QA-platforms and IT-infrastructure for data collection with different formats. In addition, EPTN-WP1 will define the requirements for high quality clinical trials and set up an infrastructure for methodological support.

\section{References}

[1] Leroy R, Benahmed N, Hulstaert F, et al. Hadron therapy in children: An update of the scientific evidence for paediatric cancers. KCE Reports 235. D/2015/10.273/04 ed. Brussels: Health Technology
Assessment Brussels: 2015.

[2] Luhr A, von Neubeck C, Krause M, Troost EGC. Relative biological effectiveness in proton beam therapy - current knowledge and future challenges (accepted for publication in Clinical and Translational Radiation Oncology).

[3] Proton radiotherapy. Horizon scanning report. Den Haag, Health Council of the Netherlands, December 14, 2009 (pdf available on http://www.gezondheidsraad.nl).

[4] KNAW (2014). Evaluation of new technology in health care. In need of guidance for relevant evidence. Amsterdam, KNAW (pdf available on http://www.knaw.nl).

[5] Widder J, van der Schaaf A, Lambin P, Marijnen CA, Pignol JP, Rasch CR, et al. The quest for evidence for proton therapy: model-based approach and precision medicine. Int J Radiat Oncol Biol Phys
2011;95:30-6.

[6] Langendijk JA, Lambin P, De Ruysscher D, Widder J, Bos M, Verheij M. Selection of patients for radiotherapy with protons aiming at reduction of side effects: the model-based approach. Radiother Oncol 2013;107:267-73.

[7] Vergouwe $Y$, Nieboer D, Oostenbrink R. A closed testing procedure to select an appropriate method for updating prediction models. Stat Med 2016;36:4529-39.

[8] Beetz I, Schilstra C, van Luijk P. External validation of three dimensional conformal radiotherapy based NTCP models for patient-rated xerostomia and sticky saliva among patients treated with intensity modulated radiotherapy. Radiother Oncol 2012;105:94-100.

[9] Lustberg T, van Soest J, Gooding M, Peressutti D, Aljabar P, van der Stoep J, et al. Clinical evaluation of atlas and deep learning based automatic contouring for lung cancer. Radiother Oncol 2018;126:3127.

[10] Weber DC, Hurkmans CW, Melidis C, Budach W, Langendijk JH, Peters L, et al. Outcome impact and cost-effectiveness of quality assurance for radiotherapy planned for the EORTC 22071-24071 prospective study for head and neck cancer. Radiother Oncol 2014;111:393-9.

[11] Relton C, Torgerson D, O'Cathain A, Nicholl J. Rethinking pragmatic randomized controlled trials: introducing the 'cohort multiple randomized controlled trial' design. BMJ 2010;340:c1066.

[12] Weber DC, Abrunhosa-Branquinho A, Bolsi A, Kacperek A, Dendale R, Geismar D, et al. Profile of European proton and carbon ion therapy centers assessed by the EORTC facility questionnaire.
Radiother Oncol 2017;124:185-9. 
[13] Weber DC, Poortmans PM, Hurkmans CW, Aird E, Gulyban A, Fairchild A. Quality assurance for prospective EORTC radiation oncology trials: the challenges of advanced technology in a multicenter international setting. Radiother Oncol 2011;100:150-6.

[14] Fairchild A, Aird E, Fenton PA, Gregoire V, Gulyban A, Lacombe D, et al. EORTC Radiation Oncology Group quality assurance platform: establishment of a digital central review facility. Radiother Oncol
2012;103:279-86.

[15] Hamel JF, Saulnier P, Pe M, Zikos E, Musoro J, Coens C, et al. A systematic review of the quality of statistical methods employed for analyzing quality of life data in cancer randomised controlled trials.
Eur J Cancer 2017;83:166-76. 
Table 1

iummary of different levels of datasets.

\begin{tabular}{|c|c|c|c|}
\hline Level & Description & Status & Items \\
\hline Level I & Minimal dataset & Mandatory & $\begin{array}{l}\text { Minimal baseline variables } \\
\text { Treatment characteristics } \\
\text { Patient-reported outcomes (PROMs) } \\
\text { Minimal set of toxicity items } \\
\text { Utilities }\end{array}$ \\
\hline Level II & $\begin{array}{l}\text { More extensive dataset but still } \\
\text { considered standard of care }\end{array}$ & Optional & $\begin{array}{l}\text { Tumour control and survival } \\
\text { Extensive sets of baseline variables } \\
\text { Additional PROMs } \\
\text { Extensive set of toxicity items } \\
\text { 3D dose distributions, including planning CT-scans, } \\
\text { DICOM-RT-struct and DICOM-RT-dose }\end{array}$ \\
\hline Level III & $\begin{array}{l}\text { More extensive dataset not considered standard } \\
\text { of care and requiring medical ethical approval }\end{array}$ & Optional & $\begin{array}{l}\text { Additional diagnostic procedures at baseline or follow up. } \\
\text { e.g. imaging, bio-samples, function tests }\end{array}$ \\
\hline
\end{tabular}


Table 2

Overview of level I generic dataset for all patients irespective of tumour site

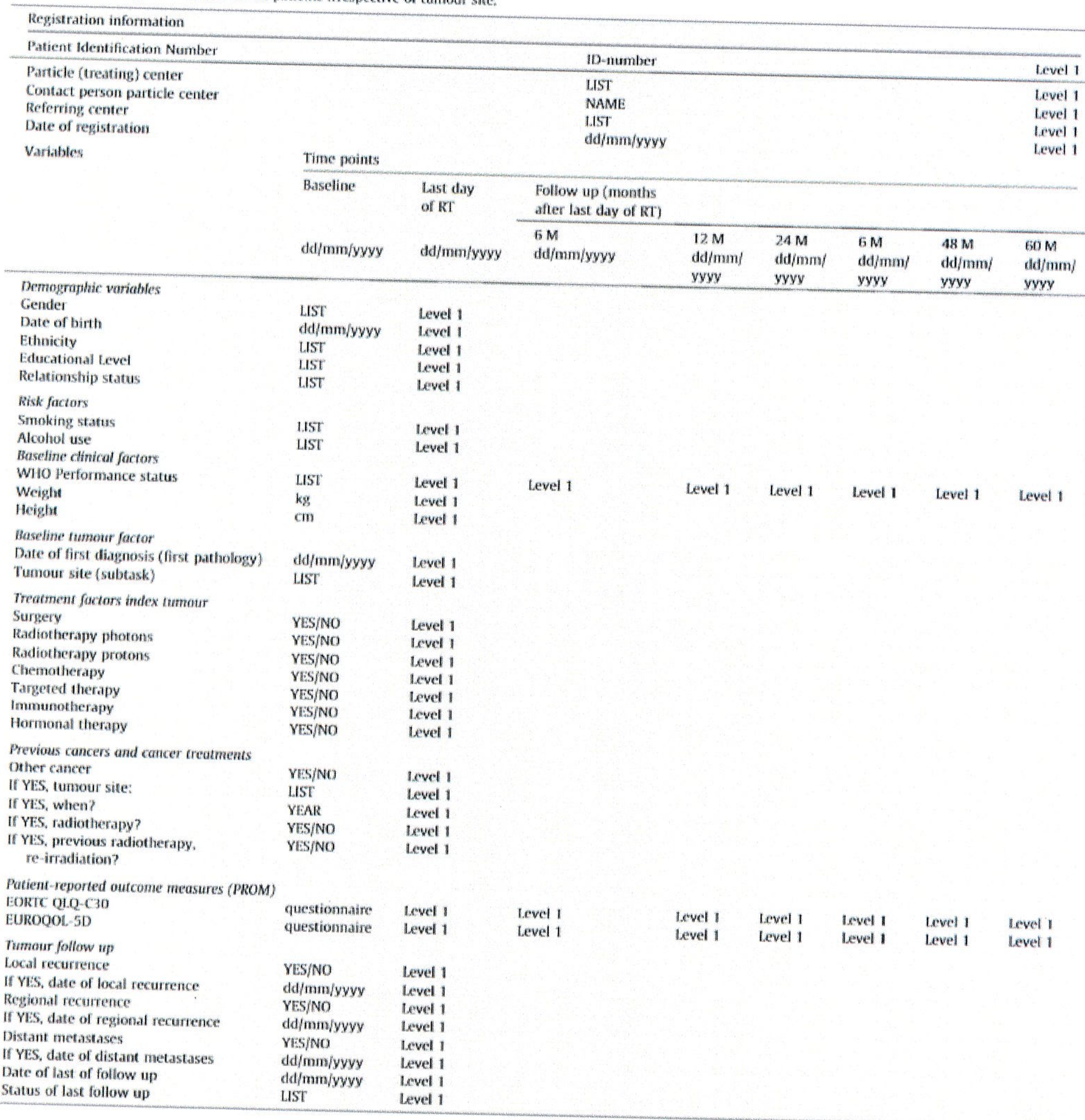

